\title{
MODIFICAÇÃO DE ESCÓRIA DE ACIARIA FUNDIDA PARA APLICAÇÃO EM CIMENTO*
}

\author{
João Batista Ferreira Neto ${ }^{1}$ \\ Catia Fredericci \\ João Oswaldo Macedo Garcia de Faria ${ }^{3}$ \\ Fabiano Ferreira Chotoli ${ }^{4}$ \\ Tiago Ramos Ribeiro ${ }^{5}$ \\ Valdecir Angelo Quarcioni ${ }^{6}$
}

\section{Resumo}

A escória de aciaria pode ser uma alternativa como mistura para o cimento portland, substituindo parcialmente a escória de alto-forno. Um processo pirometalúrgico tem sido desenvolvido para promover a modificação química da escória de aciaria LD. Os experimentos foram realizados através da refusão de $300 \mathrm{~kg}$ de escória de aciaria LD, seguido de sua modificação no estado líquido. Essas escórias foram resfriadas com o uso de esferas metálicas. Análises de fluorescência de raios- $X$ (FRX) e microscopia eletrônica de varredura (MEV) das escórias revelaram uma relação entre composição química, condição de resfriamento e a formação de fases cristalina ou vítrea. As amostras de cimento foram obtidas através da mistura de $25 \%$ de escória modificada e $75 \%$ de cimento Portland, resultando em mais de 280 $\mathrm{J} / \mathrm{g}$ de calor de hidratação acumulado em $72 \mathrm{~h}$, expansão abaixo de $0,1 \%$ em ensaio em autoclave e resistências à compressão acima de $42 \mathrm{MPa}$ com 28 dias. $\mathrm{O}$ processo mostra potencial para ser aplicado no tratamento de escória de aciaria LD.

Palavras-chave: Escória; Cimento; Modificação; Rejeitos.

\section{TREATMENT OF MOLTEN STEEL SLAG FOR CEMENT APPLICATION Abstract}

Steel slag can be an alternative in cement mineral admixture, partially as a substitute for blast furnace slag. A pyrometallugical process has been investigated to promote the chemical composition modification of molten steelmaking slag in a metallurgical reactor. Experiments were conducted by remelting $300 \mathrm{~kg}$ of steel slag followed by chemical modification of liquid slag. The modified slags were naturally cooled in the metallurgical reactor or cooled by steel balls. XRD and SEM analysis of slag samples revealed the relationship among chemical composition, cooling conditions and amorphous and crystalline phases. Cement samples were produced by mixing $25 \%$ of treated steelmaking slag with $75 \%$ of Portland cement, resulting in more than 280 $\mathrm{J} / \mathrm{g}$ of accumulated hydration heat in $72 \mathrm{~h}$, expansion lower than $0,1 \%$ in the autoclave tests and compressive strength higher than $42 \mathrm{MPa}$ after 28 days. The process indicates potential to be applied as a steelmaking slag treatment.

Keywords: Slag; Cement; Modification; Waste.

1 Engenheiro Metalurgista, Doutor, Pesquisador, Laboratório de Processos Metalúrgicos, Instituto de Pesquisas Tecnológicas, São Paulo, São Paulo, Brasil.

2 Química, Doutora, pesquisadora, Laboratório de Materiais de Construção Civil, Instituto de Pesquisas Tecnológicas, São Paulo, São Paulo, Brasil.

3 Engenheiro Metalurgista, Bacharel, pesquisador, Laboratório de Processos Metalúrgicos, Instituto de Pesquisas Tecnológicas, São Paulo, São Paulo, Brasil.

4 Químico, Mestre, pesquisador, Laboratório de Materiais de Construção Civil, Instituto de Pesquisas Tecnológicas, São Paulo, São Paulo, Brasil.

5 Engenheiro Metalurgista, Mestre, pesquisador, Laboratório de Processos Metalúrgicos, Instituto de Pesquisas Tecnológicas, São Paulo, São Paulo, Brasil.

6 Químico, Doutor, pesquisador, Laboratório de Materiais de Construção Civil, Instituto de Pesquisas Tecnológicas, São Paulo, São Paulo, Brasil. 


\section{INTRODUÇÃO}

A indústria da construção no Brasil tem crescido mais de $5 \%$ ao ano, enquanto a produção anual bruta de aço está estagnada em aproximadamente 32 milhões de toneladas nos últimos 10 anos, sem nenhuma expectativa de crescimento. Portanto, a expectativa é que falte escória de alto-forno num futuro breve. A escória de aciaria poderia ser uma alternativa como adição mineral no cimento, como substituição à escória de alto-forno [1-4]. Porém, o uso direto da escória de aciaria no cimento não é recomendado devido à sua expansibilidade durante a hidratação, causada pelo $\mathrm{CaO}$ e $\mathrm{MgO}$ livres e pela fase $\mathrm{RO}$ rica em Mg [5,6]. A atividade hidráulica das escórias é afetada pela composição química, teor de fase vítrea e pela combinação de ambos [7]. Escórias com basicidade elevada geralmente são cristalinas, enquanto estruturas vítreas são observadas em escórias acidas com teores suficientes de $\mathrm{Al}_{2} \mathrm{O}_{3}$. Além disso, a formação de fase vítrea ou cristalina depende das condições de resfriamento [8]. Este trabalho tem como objetivo o desenvolvimento de um processo pirometalúrgico com modificação da composição da escória líquida, com rejeitos ricos em $\mathrm{SiO}_{2}$ e $\mathrm{Al}_{2} \mathrm{O}_{3}$, na tentativa de obtenção de fase vítrea no resfriamento da escória para adequá-la como mistura ao cimento.

\section{MATERIAIS E MÉTODOS}

As escórias foram modificadas em um reator pirometalúrgico, através da adição de agentes modificadores em $300 \mathrm{~kg}$ de escória de aciaria LD líquida. Para a modificação foram utilizados dois subprodutos industriais, um rico em alumina e outro rico em sílica.

Depois de modificadas, as escórias foram resfriadas com esferas de aço, utilizando o processo desenvolvido e patenteado pela Paul Wurth, que autorizou o Instituto de Pesquisas Tecnológicas (IPT), com base em um acordo de cooperação, a realizar os testes usando tais condições de resfriamento. O principio do resfriamento com esferas está descrito em patente [11] e na literatura [12]. Houve um aumento da relação massa de esfera/massa de escória ( $\mathrm{M}_{\mathrm{es}} / \mathrm{M}_{\mathrm{esc}}$ ) de aproximadamente $17 \%$ no teste SS-M7 e 60\% no teste SS-M8 em relação ao teste da escória SS-M4. A escória SS foi analisada como recebida, ou seja, resfriada na indústria siderúrgica. Depois do resfriamento, as esferas de aço foram separadas da escória por separação magnética. As escórias foram moídas e homogeneizadas para obter amostras representativas para a caracterização química e mineralógica. Simulações termodinâmicas, realizadas no FactSageTM 6.4, foram utilizadas para prever as fases formadas durante a solidificação das escórias.

As escórias modificadas foram cominuídas até partículas menores que $0,075 \mathrm{~mm}$, e misturadas com cimento Portland comum, numa proporção de 25\%/75\%(escória modificada/cimento Portland), identificadas como CPM-MX. Foi preparado ainda, um cimento com escória de aciaria LD para comparação. Ensaios de resistência à compressão foram executados, de acordo com a NBR 7215/96, com as faixas de valores preconizadas na NBR 11578 de acordo com a NBR 11578, também foram feitos ensaios de expansão em autoclave (ASTM C 151 2005) nas amostras (CPM). A atividade hidráulica foi determinada através da medição do calor gerado durante a hidratação no período de 72 horas.

A Tabela 1 mostra a composição química da escória de aciaria in natura (SS) e das outras três escórias modificadas (SS-MX), determinadas através de fluorescência de raios-X (FRX). 
Tabela 1. Composição química e basicidade $\left(\mathrm{CaO} / \mathrm{SiO}_{2}\right)$ da escória de aciaria (SS) e escórias modificadas (\% em peso)

\begin{tabular}{c|c|c|c|c}
\hline & SS & SS-M4 & SS-M7 & SS-M8 \\
\hline $\mathrm{Fe}_{2} \mathrm{O}_{3}$ & 13,6 & 2,0 & 0,5 & 3,9 \\
\hline $\mathrm{FeO}$ & 18,3 & 7,6 & 3,8 & 6,3 \\
\hline $\mathrm{Fe}^{\circ}$ & 0,2 & 0,3 & 0,3 & 0,5 \\
\hline $\mathrm{CaO}$ & 38,1 & 37,3 & 37,2 & 33,6 \\
\hline $\mathrm{S}$ & 0,1 & $<0,1$ & 0,06 & 0,04 \\
\hline $\mathrm{SiO}_{2}$ & 9,9 & 32,2 & 30,8 & 31,8 \\
\hline $\mathrm{Al}_{2} \mathrm{O}_{3}$ & 1,7 & 4,8 & 11,6 & 10,7 \\
\hline $\mathrm{MgO}$ & 8,6 & 9,8 & 9,5 & 9,8 \\
\hline $\mathrm{TiO}_{2}$ & 0,3 & 0,2 & 0,3 & 0,3 \\
\hline $\mathrm{MnO}$ & 4,4 & 3,1 & 3,3 & 3,8 \\
\hline $\mathrm{P}_{2} \mathrm{O}_{5}$ & 1,3 & 0,8 & 0,7 & 0,9 \\
\hline $\mathrm{CaOlivre}$ & 5,7 & $\mathrm{ND}$ & 0,2 & 0,2 \\
\hline $\mathrm{B}\left(\mathrm{CaO} / \mathrm{SiO}_{2}\right)$ & 3,85 & 1,16 & 1,21 & 1,06 \\
\hline $\mathrm{FeO}+\mathrm{Fe}_{2} \mathrm{O}_{3}$ & 31,9 & 9,6 & 4,3 & 10,2 \\
\hline
\end{tabular}

$\left(^{*}\right)$ FeO: ASTM E 246-10 - Determination of Iron by Dichromate Titrimetry.

$\left.{ }^{(* *}\right) \mathrm{Fe}^{0}: \mathrm{XU}, \mathrm{Z}$ et al[10] $\left(^{(* \star}\right) \mathrm{Fe}^{3+}(\%)=\mathrm{Fet}_{\mathrm{t}} \mathrm{FRX}(\%)-\mathrm{Fe}^{2+}(\%)-\mathrm{Fe}^{0}$.

\section{RESULTADOS E DISCUSSÃO}

A Tabela 2 apresenta as fases mineralógicas encontradas na escória de aciaria LD e nas escórias de aciaria LD modificadas, determinadas por difração de raios- $\mathrm{X}$, adotando a metodologia Rietveld para quantificação. Conforme apresentado na Tabela 1, a escória de aciaria (SS) é totalmente cristalina, pois esse tipo de escória tem alta basicidade $\left(\mathrm{CaO} / \mathrm{SiO}_{2}=3,85\right)$ e um alto percentual de óxidos de ferro, que pode atuar como núcleo para crescimento de cristais [13]. As fases cristalinas são aquelas tipicamente observadas nas escórias de aciaria $[14,4,8]$ : brownmilerita $\left(\mathrm{Ca}_{2}(\mathrm{Fe}, \mathrm{Al})_{2} \mathrm{O}_{5}\right)$, larnita $\left(\beta-\mathrm{Ca}_{2} \mathrm{SiO}_{4}\right)$, fase $\mathrm{RO}$ (solução sólida entre $\mathrm{FeO}, \mathrm{MnO}, \mathrm{MgO}$ e $\mathrm{CaO}$ ) e $\mathrm{CaO}$ livre. O teor de $\mathrm{CaO}$ livre $(4,2 \%)$ é muito alto para garantir estabilidade volumétrica da escória. A composição química da fase $\mathrm{RO}$, determinada através de espectroscopia de energia dispersiva (EDS) em diferentes pontos da microestrutura da escória de aciaria, mostrou uma alta relação molar $\mathrm{MgO} / \mathrm{FeO}$ $(0,81)$, que junto com o $\mathrm{CaO}$ e o $\mathrm{MgO}$ livre, é uma limitação para a utilização dessa escória no cimento. Qian et al. [6] relataram que relações $\mathrm{MgO} / \mathrm{FeO}$ mais elevadas, aumentam o potencial de reatividade com a água, afetando a expansão do material. De acordo com os resultados, a fase RO da escória SS é fase wustita rica em Mg, que possui potencial para reagir com a água formando brucita $\left(\mathrm{Mg}(\mathrm{OH})_{2}\right)$. 
Tabela 2. Fases mineralógicas (\% em peso) da escória de aciaria e escórias modificadas, determinadas por DRX (Rietveld)

\begin{tabular}{|c|c|c|c|c|}
\hline Fases & SS & SS-M4 & SS-M7 & SS-M8 \\
\hline $\begin{array}{c}\text { Larnita } \\
\left(\mathrm{Ca}_{2} \mathrm{SiO}_{4}\right)\end{array}$ & 35,6 & - & & \\
\hline $\begin{array}{l}\text { Brownmlilerita } \\
\mathrm{Ca}_{2}(\mathrm{Al}, \mathrm{Fe})_{2} \mathrm{O}_{5} \\
\end{array}$ & 38,7 & - & & \\
\hline $\begin{array}{c}\mathrm{RO} \\
(\mathrm{FeO}, \mathrm{MgO}, \mathrm{MnO}, \mathrm{CaO})\end{array}$ & 21,5 & - & & \\
\hline $\begin{array}{l}\mathrm{Cal} \\
\mathrm{CaO}\end{array}$ & 4,2 & - & & \\
\hline $\begin{array}{c}\text { Monticelita } \\
\mathrm{CaO}_{\mathrm{MgO} . \mathrm{SiO}_{2}}\end{array}$ & - & 42,8 & & \\
\hline $\begin{array}{c}\text { Merwinita } \\
\text { 3CaO.MgO. } \mathrm{SiO}_{2}\end{array}$ & - & 34,9 & 31,1 & 33,7 \\
\hline $\begin{array}{c}\text { Akermanita } \\
2 \mathrm{CaO} . \mathrm{MgO} . \mathrm{SiO}_{2}\end{array}$ & - & 10,9 & 8,8 & \\
\hline $\begin{array}{c}\text { Gehlenita } \\
2 \mathrm{CaO} \cdot \mathrm{Al}_{2} \mathrm{O}_{3} \cdot \mathrm{SiO}_{2}\end{array}$ & - & 1,1 & 5,3 & \\
\hline $\begin{array}{c}\text { Melilite } \\
\text { (ss Ak + Ge) }\end{array}$ & - & & & 1,6 \\
\hline Vítrea & - & 11,9 & 54,3 & 62,5 \\
\hline
\end{tabular}

ss =solução sólida; Ak =akermanita e Geh = Gehlenita

A Figura 1 mostra a simulação de resfriamento da escória SS obtida pelo software FactSage $^{T M}$ 6.4, mostrando que as fases cristalinas são aquelas determinadas Por difração de raios-X, como já havia sido demonstrado na literatura [15].

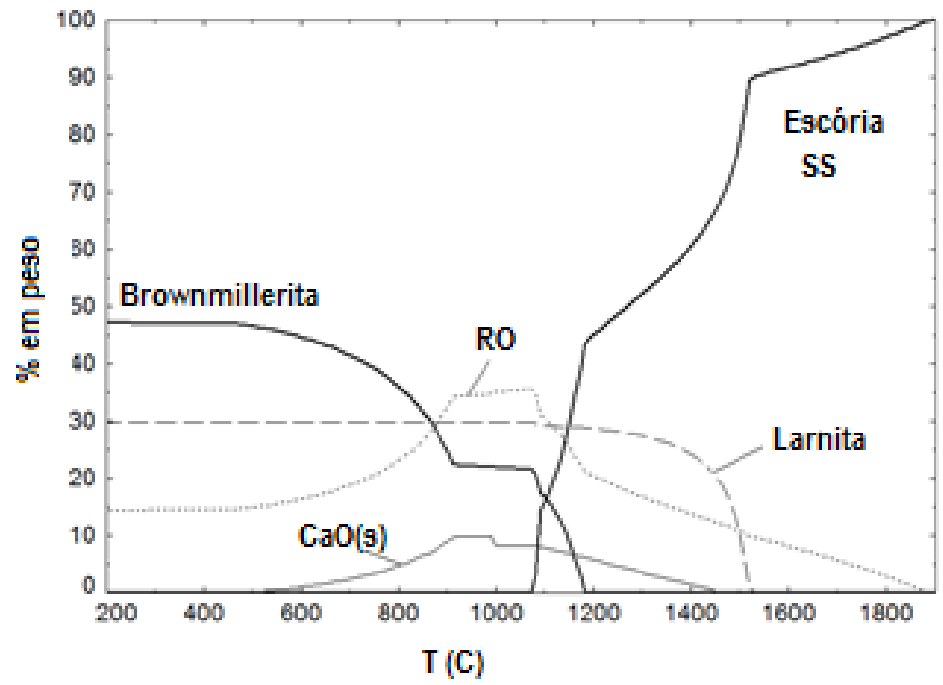

Figura 1 - Simulação termodinâmica da escória SS obtida por FactSage 6.4

Conforme apresentado na Tabela 1, todas as escórias modificadas desse grupo apresentaram baixa basicidade, entre 1,1 e 1,2 , e teores de alumina de aproximadamente $11 \%$, exceto a escória SS-M4, que apresentou $4,8 \%$ de $\mathrm{Al}_{2} \mathrm{O}_{3} . \mathrm{A}$ escória SS-M7 apresentou maior redução pelos agentes modificadores, resultando em baixo $\mathrm{FeO}+\mathrm{Fe}_{2} \mathrm{O}_{3}(4,2 \%)$ comparada com as escórias modificadas SS-M4, e SS-M8, que apresentaram, respectivamente, 9,6 e 10,2\% de $\mathrm{FeO}+\mathrm{Fe}_{2} \mathrm{O}_{3}$.

$\mathrm{Na}$ Tabela 2, observa-se que as fases cristalinas são constituídas principalmente por silicatos de cálcio e magnésio, como Merwinita, Monticelita, Akermanita e Melilita 
(solução sólida entre Akermanita e Gehlenita). Uma significativa redução da fase RO também foi observada, causada em parte pela diminuição dos teores de óxidos de ferro, devido à adição dos agentes modificadores, e parte pela transferência do $\mathrm{MgO}$ da fase RO para os silicatos de cálcio e magnésio. Além da estabilização do MgO como silicatos de cálcio e magnésio, foi observada a eliminação do $\mathrm{CaO}$ livre típico das escórias de aciaria, evitando a expansão dessas escórias.

Apesar da baixa basicidade da escória SS-M4, somente $11,5 \%$ de fase amorfa foi encontrada. Dois fatores podem explicar esse resultado, o baixo teor de alumina comparado com o das outras escórias do mesmo grupo, que pode reduzir a possibilidade da formação de fase amorfa e um resfriamento insuficiente para promover a estabilização da fase vítrea da escória.

Com objetivo de aumentar a quantidade de fase vítrea, a escória SS-M7 foi produzida com um teor de alumina maior $(11,6 \%)$ e adotando um resfriamento mais rápido do que o adotado para o resfriamento da escória SS-M4, aumentando a relação Mesf/Mesc em aproximadamente 17\%. Como mostrado na Tabela 2, foi observado um aumento de fase vítrea para $54 \%$.

A simulação da solidificação da escória SS-M7 em condições de equilíbrio termodinâmico ilustrada na Figura 2 é relativamente consistente com os resultados experimentais. A escória resfriada por esferas de aço corresponde à temperatura $1280^{\circ} \mathrm{C}$, com aproximadamente $57 \%$ em peso de fase amorfa, mostrando também a formação de Melilita (Akermanita + Gehlenita) e Merwinita, sendo que essas fases foram observadas na escória SS-M7 (Tabela 2), e são fases típicas de escória de alto-forno [15].

A escória SS-M8 foi resfriada com Mesf/Mesc bem superior à SS-M4, com aumento dessa relação de aproximadamente $60 \%$. Apesar de apresentar teor mais elevado de $\mathrm{FeO}+\mathrm{Fe}_{2} \mathrm{O}_{3}(10,2 \%)$ em relação a SS-M7 (4,3\%), que poderia atuar como sítios de nucleação para crescimento de cristais, a taxa de resfriamento foi maior, compensando a aumento no teor de ferro, e favorecendo a formação de fase vítrea, que foi de $62,5 \%$ em peso.

A Figura 3 apresenta micrografias obtidas por MEV de duas regiões de uma amostra SS-M7, sendo uma delas a região em contato com a bola de aço (Figura 3a) indicando a formação de fase vítrea, e outra correspondente a região distante do contato da bola, indicando a formação de fases cristalinas.

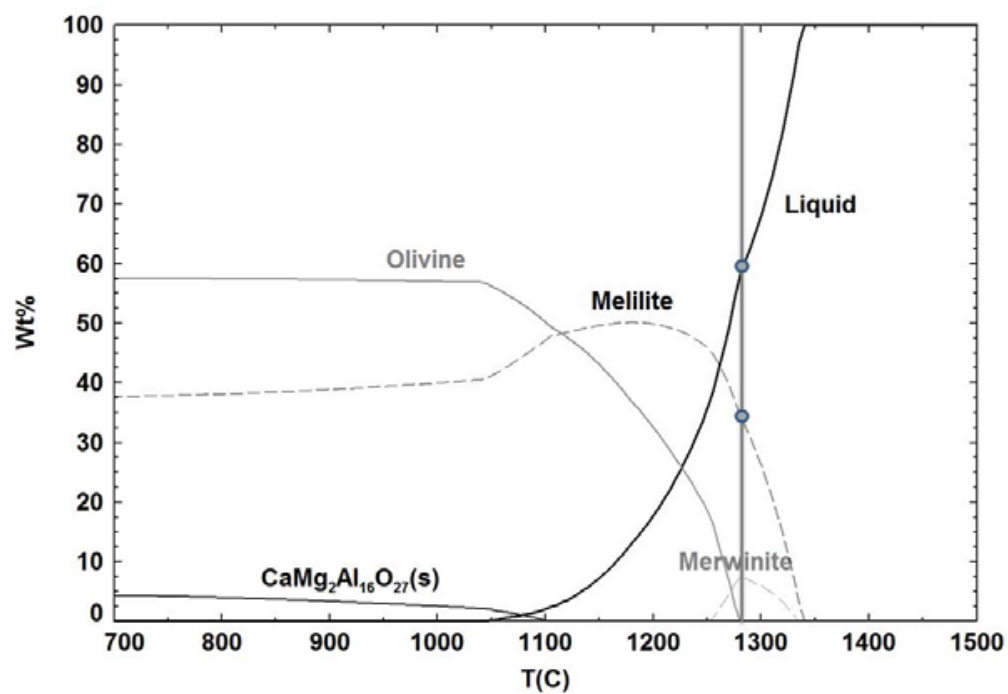

Figura 2. Simulação do resfriamento da escória SS-M7 em condições de equilíbrio termodinâmico, realizada no software FactSage ${ }^{\mathrm{TM}}$. 

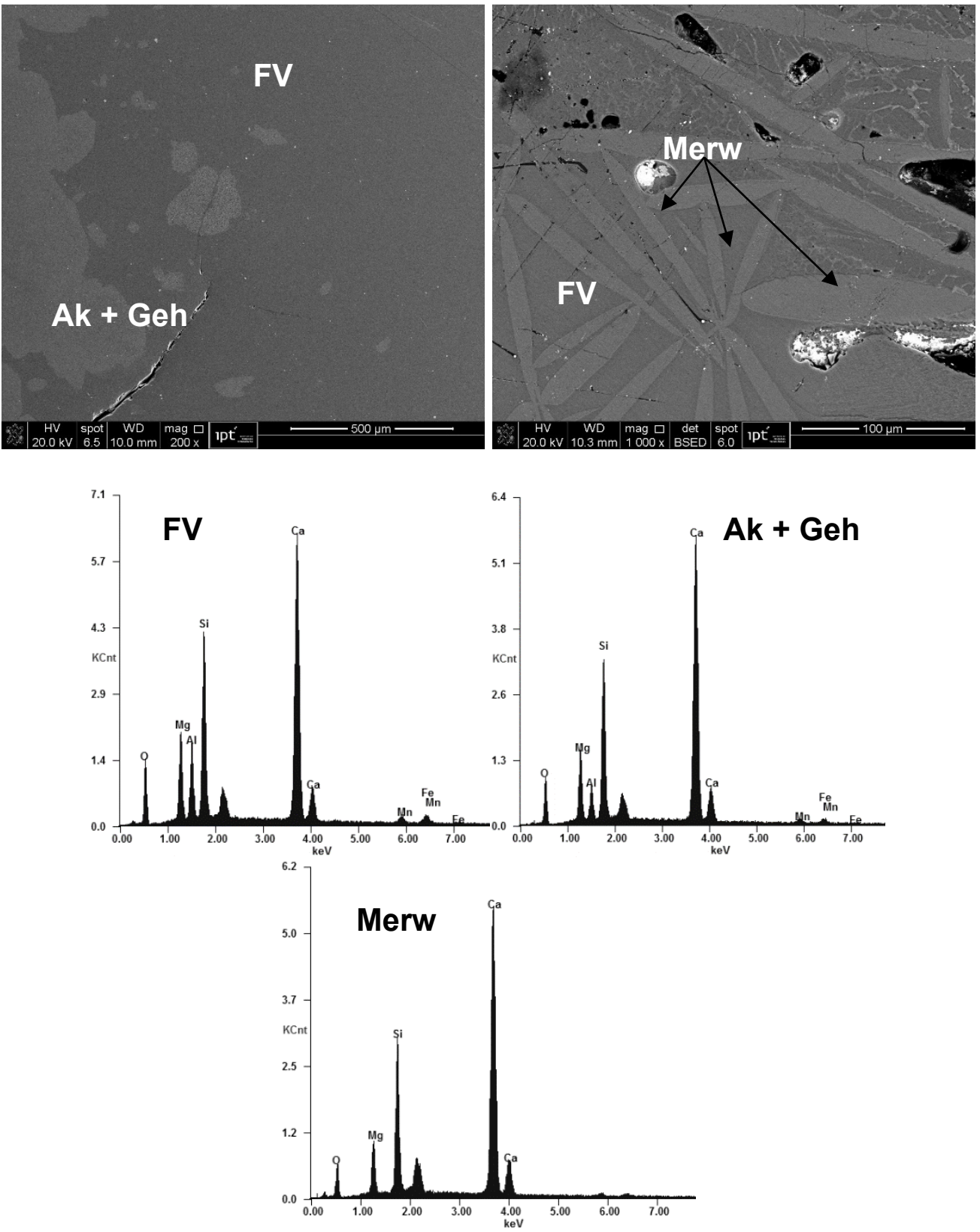

Figura 3: (a) região da amostra SS-M7 em contato com a bola de aço e (b) região amis afastada em relação ao contato com as bolas de aço. $F V=$ fase vítrea, $A k+G e h=$ Akermanita + Gehlenita e Merw=Merwinita

A Tabela 3 mostra o calor de hidratação gerado em 72 h, expansão determinada em ensaio em autoclave e resistência a compressão nas idades de 3, 7 e 28 dias para as amostras de cimento produzidas com $75 \%$ de cimento Portland ordinário e $25 \%$ de escória de aciaria (CPM-MX) e $75 \%$ de cimento Portland ordinário e $25 \%$ das escórias de aciaria modificadas. 
Tabela 3 - Calor gerado em $72 \mathrm{~h}$, expansão em autoclave e resistência a compressão em 3, 7 e 28 dias das amostras de cimento produzidas com $25 \%$ de escória e $75 \%$ de cimento Portland ordinário.

\begin{tabular}{c|c|c|c|c|c}
\cline { 2 - 6 } & $\begin{array}{c}\text { Calor } \\
\text { Hidratação } \\
(72 \mathrm{~h}) \mathrm{J} / \mathrm{g}\end{array}$ & $\begin{array}{c}\text { Expansão em } \\
\text { Autoclave }(\%)\end{array}$ & $\mathrm{RM}(3 \mathrm{~d})$ & $\mathrm{RM}(7 \mathrm{~d})$ & $\mathrm{RM}(28 \mathrm{~d})$ \\
\hline CPM-SS & 280,5 & 0,44 & 27,0 & 34,1 & 41,1 \\
\hline CPM-M4 & 278,2 & 0,07 & 28,3 & 31,2 & 36,9 \\
\hline CPM-M7 & 307,0 & 0,04 & 28,0 & 32,9 & 43,8 \\
\hline CPM-M8 & 284,3 & 0,12 & 30,2 & 37,1 & 45,3 \\
\hline
\end{tabular}

$\mathrm{RM}=$ resistência mecânica à compressão, $\mathrm{d}=$ dia

A Tabela 3 mostra que os calores gerados na maioria das amostras, na média, são muito próximos. Um dado interessante na Tabela 3 é o resultado de expansão em autoclave, pois os cimentos produzidos pela adição de escórias modificadas apresentaram expansões bem menores que a determinada para a amostra de cimento com escória de aciaria sem nenhuma adição, indicando que $\mathrm{CaO}, \mathrm{MgO}$ não estão livres e sim foram incorporados nas fases de silicatos cristalinos. Esse resultado indica que proporções maiores que $25 \%$ em peso podem apresentar potencial para a substituição de OCP em composições de cimentos.

As amostras de cimento produzidas com a adição das escórias SS-M7 e SS-M8 apresentaram na média maiores resistências à compressão que o cimento produzido com a adição da escória SS-M4 e SS, principalmente para 28 dias, e esse resultado pode estar relacionado com a maior quantidade de fase vítrea obtida nas amostras SS-M7 e SS-M8 ( $54 \%$ e $62 \%$, respectivamente). A resistência à compressão medida após 3,7 e 28 dias foi de 28,3, 31,2, e 36,9 MPa para amostra CPM-M4 correspondendo a classe 32 , e 30,2, 37,1 e 45,3 para a amostra CPM-M8 correspondendo a classe 40 de acordo com a norma NBR 11578 (similar à EN 1971).

\section{CONCLUSÃO}

As principais conclusões são as seguintes:

- escórias com basicidades mais baixas $(1,1$ a 1,2$)$ próximas às da escória de altoforno, quando resfriadas em esferas de aço resultam na formação de fase vítrea;

- o teor de fase vítrea está intimamente relacionado com a relação massa de esfera de aço/massa de escória;

- MgO e CaO livres foram estabilizados em silicatos de cálcio e magnésio através do tratamento da escória líquida.

- as amostras de cimento compostas por $25 \%$ de escórias modificadas, contendo maior fração de fase vítrea, apresentaram na média, um aumento da resistência à compressão se comparadas com as amostras de cimento com $25 \%$ de escória de aciaria LD, principalmente após 28 dias;

-todas as amostras de cimento com escórias modificadas apresentaram uma expansões expressivamente menores do que a expansão observada no cimento moldado com escória de aciaria LD.

\section{Agradecimentos}

Os autores agradecem o suporte financeiro da InterCement and Embrapii. 


\section{REFERÊNCIAS}

1 Belhadj E, Diliberto C, Lecomte A. Characterization and activation of Basic Oxygen Furnace slag. Cement and Concrete. 2012; 34: 34-40.

2 Faraone N, Tonello G, Furlani E, Maschio S. Steelmaking slag as aggregate for mortars: Effects of particle dimension on compression strength. Chemosphere. 2009; 77: $1152-1156$.

3 Li JX, Yu QJ, Wei JX, Zhang TS. Structural characteristics and hydration kinetics of modified steel slag. Cement and Concrete. 2011; 41: 324-329.

4 Tossavainen M, Engstrom F, Yang Q, Menad N, Larsson ML, Bjorkman B. Characteristic of steel slag under different cooling conditions. Waste Management. 2007; 27: 1335-1344.

$5 \quad$ Kriskova L, Pontikes Y, Pandelaers L, Cizer O, Jones PT, van Balen K, Blanpain B. Effect of High Cooling Rates on the Mineralogy and Hydraulic Properties of Stainless Steel Slags. Metallurgical and Materials Transaction B. 2013; 44: 1173-1184.

6 Qian GR; Sun DD; Tay JH; Lai ZY. Hydrothermal reaction and autoclave stability of Mg bearing RO phase in steel slag - British Ceramic Transactions. 2002; 101, 4: 159-164.

7 Mostafa NY, El-Hemaly SAS, Al-Wakeel El, El-Korashy SA, Brown PW.

Characterization and evaluation of hydraulic activity of water-cooled slag and air-cooled slag. Cement and Concrete Research. 2001; 31: 899-904.

8 Gautier M, Poirier J, Bodenan F, Franceschini G, Véron E. Basic oxygen furnace (BOF) slag cooling: Laboratory characteristics and prediction calculations. International Journal of Mineral Processing. 2013; 123: 94-101.

9 Ferreira Neto JB, Ribeiro TR, Lotto AA, Quarcioni VA, Chotoli FF. Sistema de Modificação de Escória, Patent application BR 102014023505 1; 2014.

10 Xu Z, Hwang J, Greenlund R, Huang X, Luo L, Anschuetz S. Quantitative Determination of Metallic Iron Content in Steel-Making Slag". Journal of Minerals and Materials Characterization and Engineering. 2003; 2: 65-70.

11 Solvi M, Greiveldinger B, Hoffmann M, Friederici C, Michels D. Granulation of metallurgical slag. WO2012/0836 A1. 2012; pp 1-15.

12 Kappes H, Michels D. Dry slag granulation and energy recovery. In: Proceedings of the fourth international slag valorization symposium. Leuven, 2015; pp 39-52.

13 Jung SS, Sohn II. Effect of FeO Concentration on the Crystallization of HighTemperature CaO-Al2O3-MgO-FeO Melts. Journal American Ceramic Society. 2013; 96, 4: 1309-1316.

14 Shi CJ. Steel Slag - Its Production, Processing, Characteristics and Cementitious Properties. Journal of Materials in Civil Engineering. 2004; 16: 230-236.

15 Ferreira Neto, J. B.; Faria, J. O. G. ; Fredericci, C.; Chotoli, F. F.; Silva, A. L. N.; Ferraro, B. B.; Ribeiro, T. R. ; Malynowskyj, A.; Quarcioni, V. A. ; Lotto, A. A. . Modification of molten steelmaking slag for cement application. J. Sustain. Metall. DOI 10.1007/s40831-015-0031-7. 2015; 1-15.

16 Chotoli F, Martho ACR, Quarcioni VA, Castanho MA. Avaliação do perfil térmico de calorímetro de condução isotérmico para estudos de calor de hidratação de pastas de cimento Portland. Enqualab_Congresso de Qualidade em Metrologia. 2013; pp 1-6.

17 American Society for Testing Materials (ASTM) C 151. Autoclave expansion of hydraulic cement. 2005; pp 1-4. 